\title{
Transmuted Exponentiated Gumbel Distribution (TEGD) and its Application to Water Quality Data
}

\author{
Deepshikha Deka \\ Department of Statistics \\ North Eastern Hill University, Shillong, India \\ deepshikha.deka11@gmail.com \\ Bhanita Das \\ Department of Statistics \\ North Eastern Hill University, Shillong, India \\ bhanitadas83@gmail.com \\ Bhupen K. Baruah \\ Department of Chemistry \\ J. B. College Jorhat, Assam, India \\ bhupen_baruah2009@rediffmail.com
}

\begin{abstract}
The Transmuted Exponentiated Gumbel Distribution (TEGD) has been derived using Exponentiated Gumbel Distribution (EGD) and the Quadratic Rank Transmutation Map (QRTM). The analytical expressions and shapes of the distribution function, probability density function, hazard rate function and reliability function are studied. The parameters of the TEGD are estimated by the method of maximum likelihood. Finally the TEGD is applied to real data set of water quality parameter and found to be better fit than Exponentiated Gumbel Distribution (EGD) and Gumbel Distribution (GD).
\end{abstract}

Keywords: TEGD, QRTM, Reliability Function, Hazard Rate Function, Maximum Likelihood.

\section{Introduction}

Transmuted distributions have been discussed dynamically in frequently occurring large scale experimental statistical data for model selection and related issues. In applied sciences such as environmental, medicine, engineering etc. modeling and analyzing experimental data are essential. There are several distributions which can be used to model such kind of experimental data. The procedures used in such a statistical analysis depend heavily on the assumed probability model or distributions. That is why the development of large classes of standard probability distributions along with relevant statistical methodologies has been expanded. However, there still remain many important problems where the real data does not follow any of the classical or standard probability models.

The Gumbel Distribution (GD) is a very popular statistical distribution due to its extensive applicability in several areas and its wide applications has been reported by Kotz and Nadarajah (2000). The applicability of GD in the field of flood frequency analysis, network, space, software reliability, structural and wind engineering are reported by Cardeiro et al., (2012). Nadarajah (2006) introduced Exponentiated Gumbel 
Distribution (EGD) based on Gumbel Distribution (GD) and illustrated its applicability in the area of global warming modeling, rainfall modeling, wind speed modeling etc. Due to its wide applicability in different fields of science, the generalization of Gumbel Distribution has become important.

Now a days transmuted distributions and their mathematical properties are widely studied for applied sciences experimental data sets. Transmuted Rayleigh Distribution (Merovci, 2013), Transmuted Inverse Rayleigh Distribution (Ahmad et al., 2014), Transmuted Generalized Inverse Weibull Distribution (Khan and King, 2013), Transmuted Modified Inverse Weibull Distribution (Elbatal, 2013), Transmuted Log-logistic Distribution (Aryal, 2013), Transmuted Modified Weibull Distribution \& Transmuted Lomax Distribution (Ashour and Eltehiwy, 2013), Transmuted Frechet Distribution (Mahmoud \& Mandouh, 2013), Transmuted Pareto Distribution (Merovci \& Puka, 2014), Transmuted Generalized Gamma Distribution (Lucena et al., 2015), Transmuted Weibull Lomax Distribution (Afify et al., 2015) are reported with their various structural properties including explicit expressions for the moments, quantiles, entropies, mean deviations and order statistics. All the above transmuted distributions are derived by using Quadratic Rank Transmutation Map(QRTM) studied by Shaw \& Buckley (2007). Report reveals that some properties of these distributions along with their parameters are estimated by using maximum likelihood and Bayesian methods. Usefulness of some of these new distributions are also illustrated with experimental data sets.

Transmuted Gumbel Distribution (TGD) along with several mathematical properties has studied by Aryal and Tsokos (2009) using Quadratic Rank Transmutation Map(QRTM)and reported that TGD can be used to model climate data. Therefore an attempt has been made to developed Transmuted Exponentiated Gumbel Distribution (TEGD) using Exponentiated Gumbel Distribution (EGD) and the Quadratic Rank Transmutation Map(QRTM). The parameters of the TEGD are estimated by the method of maximum likelihood and applied to the water quality parameter data sets for study the usefulness of the model.

A random variable $X$ is said to have a transmuted distribution if its cumulative distribution function $(c d f)$ is given by

$$
G(x)=(1+\lambda) F(x)-\lambda F^{2}(x),|\lambda| \leq 1
$$

Where $G(x)$ is the $c d f$ of the transmuted distribution and $F(x)$ is the $c d f$ of the base distribution. Differentiating (1) w.r.t. X, it gives the probability density function $(p d f)$ of the transmuted distribution as

$$
g(x)=f(x)[1+\lambda-2 \lambda F(x)]
$$

Where $g(x)$ and $f(x)$ are the corresponding $p d f$ of $G(x)$ and $F(x)$ respectively. It is observed that at $\lambda=0$, we have the base distribution of the random variable $X$. 


\section{$c d f \& p d f$ of TEGD:}

For $-\infty<x<\infty,-\infty<\mu<\infty, \alpha>0$ and $\sigma>0$ the pdf and $\boldsymbol{c d f}$ of Exponentiated Gumbel Distribution (EGD) can be expressed as equation (3) \& (4) respectively

$$
\begin{aligned}
& f(x)=\frac{\alpha}{\sigma}\left[1-\exp \left\{-\exp \left(-\frac{x-\mu}{\sigma}\right)\right\}\right]^{\alpha-1} \exp \left(-\frac{x-\mu}{\sigma}\right) \exp \left\{-\exp \left(-\frac{x-\mu}{\sigma}\right)\right\} \\
& F(x)=1-\left[1-\exp \left\{-\exp \left(-\frac{x-\mu}{\sigma}\right)\right\}\right]^{\alpha}
\end{aligned}
$$

Using (1) the $c d f$ of Transmuted Exponentiated Gumbel Distribution (TEGD) for $-\infty<x<\infty,-\infty<\mu<\infty, \alpha>0, \sigma>0$ and $|\lambda| \leq 1$ is

$$
\begin{array}{r}
G(x)=1-\left\{1-\exp \left(-\exp \left(-\frac{x-\mu}{\sigma}\right)\right)\right\}^{\alpha}[1-\lambda \\
\left.+\lambda\left\{1-\exp \left(-\exp \left(-\frac{x-\mu}{\sigma}\right)\right)\right\}^{\alpha}\right]
\end{array}
$$

The corresponding $p d f$ of TEGD is

$$
\begin{aligned}
& g(x) \\
& =\frac{\alpha}{\sigma}\left\{1-\exp \left(-\exp \left(-\frac{x-\mu}{\sigma}\right)\right)\right\}^{\alpha-1}\left\{\exp \left(-\exp \left(-\frac{x-\mu}{\sigma}\right)\right)\right\}\left(\exp \left(-\frac{x-\mu}{\sigma}\right)\right)[1 \\
& \left.-\lambda+2 \lambda\left\{1-\exp \left(-\exp \left(-\frac{x-\mu}{\sigma}\right)\right)\right\}^{\alpha}\right]
\end{aligned}
$$

for $-\infty<x<\infty,-\infty<\mu<\infty, \alpha>0, \sigma>0$ and $|\lambda| \leq 1$.

Using series representation as

$$
(1+z)^{a}=\sum_{j=0}^{\infty} \frac{\Gamma(a+1)}{\Gamma(a-j+1} \frac{z^{j}}{j !}
$$

The expression (5) \& (6) can be written in mixture form by using (*) as (7) \& (8) respectively

$$
\begin{aligned}
& G(x)=1-(1-\lambda) \sum_{j=0}^{\infty} \frac{(-1)^{j} \Gamma(\alpha+1)}{\Gamma(\alpha-j+1)} \frac{v^{j}}{j !}-\lambda \sum_{j=0}^{\infty} \frac{(-1)^{j} \Gamma(2 \alpha+1)}{\Gamma(2 \alpha-j+1)} \frac{v^{j}}{j !} \\
& g(x)=\frac{\alpha}{\sigma} \sum_{j=0}^{\infty} \frac{(-1)^{j} \Gamma \alpha}{\Gamma(\alpha-j)} \frac{v^{j+1}}{j !} \log \left(\frac{1}{v}\right)\left[1-\lambda+2 \lambda \sum_{k=0}^{\infty} \frac{(-1)^{k} \Gamma \alpha}{\Gamma(\alpha-k)} \frac{v^{k}}{k !}\right] \\
& \text { Where } \quad v=\exp \left(-\exp \left(-\frac{x-\mu}{\sigma}\right)\right)
\end{aligned}
$$




\section{Graphical Representation of $p d f \& c d f$ of TEGD:}

For known values of $\mu, \sigma, \alpha$ and $\lambda$ the possible shapes of $c d f$ and $p d f$ of the TEGD are represented in Fig. 1 \& Fig. 2.

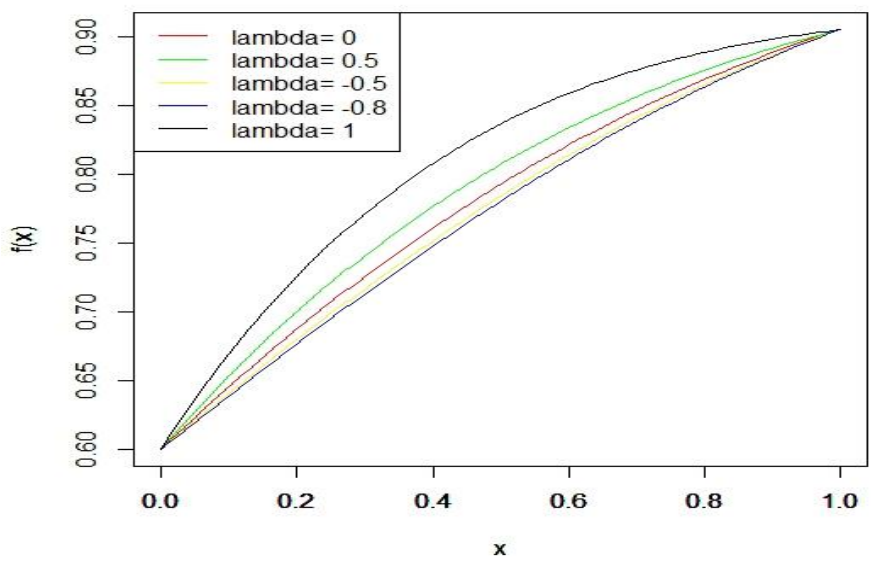

Fig.1: The $c d f$ of the TEGD for assumed values of $\alpha=2, \mu=0, \sigma=1 \& \lambda=0,0.5,-0.5,-0.8 \& 1$

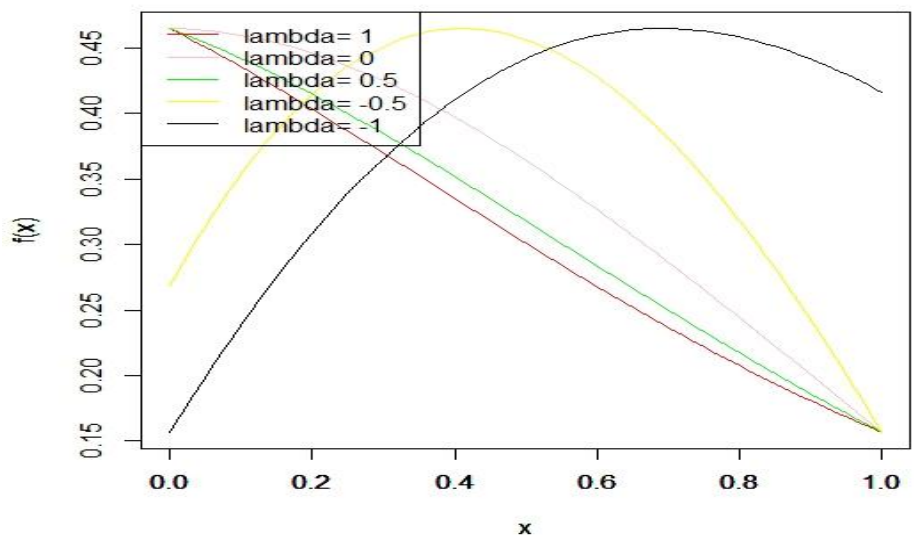

Fig.2: The $\mathrm{p} d f$ of the TEGD for assumed values of $\alpha=1, \mu=0, \sigma=1 \& \lambda=1,0,0.5,-0.5 \&-1$

\section{Moments of TEGD:}

The $\mathrm{n}^{\text {th }}$ moment $E\left(X^{n}\right)$ of a TEGD for random variable $\mathrm{X}$ can be obtained as $E\left(X^{n}\right)$

$=\frac{\alpha}{\sigma} \int_{-\infty}^{+\infty} x^{n}\left[1-\exp \left\{-\exp \left(-\frac{x-\mu}{\sigma}\right)\right\}\right]^{\alpha-1} \exp \left\{-\exp \left(-\frac{x-\mu}{\sigma}\right)\right\} \exp \left(-\frac{x-\mu}{\sigma}\right)$
$\left[1-\lambda+2 \lambda\left[1-\exp \left\{-\exp \left(-\frac{x-\mu}{\sigma}\right)\right\}\right]^{\alpha}\right] d x$ 
Setting $u=\exp \left(-\frac{x-\mu}{\sigma}\right)$ the expression reduces to

$$
\begin{aligned}
E\left(X^{n}\right)=\alpha(1-\lambda) \int_{0}^{\infty}(\mu-\sigma \log u)^{n}\{1-\exp (-u)\}^{\alpha-1} \exp (-u) d u \\
+2 \lambda \alpha \int_{0}^{\infty}(\mu-\sigma \log u)^{n}\{1-\exp (-u)\}^{2 \alpha-1} \exp (-u) d u
\end{aligned}
$$

Using binomial expression for $(a+b z)^{n}$, (9) can be written as

$$
\begin{aligned}
E\left(X^{n}\right)=\alpha(1 & -\lambda) \sum_{k_{1}=0}^{n}\left(\begin{array}{l}
n \\
k_{1}
\end{array}\right)(-\sigma)^{k_{1}} \mu^{n-k_{1}} I\left(k_{1}\right) \\
& +2 \alpha \lambda \sum_{k_{2}=0}^{n}\left(\begin{array}{l}
n \\
k_{2}
\end{array}\right)(-\sigma)^{k_{2}} \mu^{n-k_{2}} I\left(k_{2}\right)
\end{aligned}
$$

Where

$I\left(k_{1}\right)=\int_{0}^{\infty}(\log u)^{k_{1}}\{1-\exp (-u)\}^{\alpha-1} \exp (-u) d u$

and

$I\left(k_{2}\right)=\int_{0}^{\infty}(\log u)^{k_{2}}\{1-\exp (-u)\}^{2 \alpha-1} \exp (-u) d u$

Using $(*)$ we can represent $I\left(k_{1}\right)$ as

$I\left(k_{1}\right)=\sum_{l=0}^{\infty} \frac{(-1)^{l} \Gamma \alpha}{l ! \Gamma(\alpha-1)} I\left(k_{1}, l\right)$

Where, $I\left(k_{1}, l\right)$ denotes the integral

$\left(k_{1}, l\right)=\int_{0}^{\infty}(\log u)^{k} \exp \{-(l+1)\} d u$

Or $I\left(k_{1}, l\right)=\left.\left(\frac{\partial}{d a}\right)^{k}\left[(l+1)^{-a} \Gamma a\right]\right|_{a=1}$

Using $(*)$, the $I\left(k_{2}\right)$ can be expressed as

$I\left(k_{2}\right)=\sum_{l=0}^{\infty} \frac{(-1)^{l} \Gamma(2 \alpha)}{l ! \Gamma(2 \alpha-1)} I\left(k_{2}, l\right)$

Where $I\left(k_{2}, l\right)$ denotes the integral

$I\left(k_{2}, l\right)=\int_{0}^{\infty}(\log u)^{k_{2}} \exp \{-(l+1) u\} d u$

Or

$I\left(k_{2}, l\right)=\left.\left(\frac{\partial}{\partial a}\right)^{k_{2}}\left[(l+1)^{-a}, \Gamma a\right]\right|_{a=1}$ 
The expression for $I\left(k_{1}, l\right)$ and $I\left(k_{2}, l\right)$ is obtained by using Prudnikov et al. (1986).

By combining (10), (13), (15), (16) and (18), the $n^{\text {th }}$ moment of $\mathrm{X}$ can be expressed as

$$
\begin{aligned}
E\left(X^{n}\right)=\alpha \mu^{n} & (1 \\
& -\lambda) \sum_{k_{1}=0}^{n} \sum_{l=0}^{\infty} \frac{(-1)^{l+k_{1}} \Gamma \alpha \Gamma(n+1)}{k_{1} ! l ! \Gamma(\alpha-l) \Gamma\left(n-k_{1}+1\right)}\left(\frac{\sigma}{\mu}\right)^{k_{1}}\left(\frac{\partial}{\partial a}\right)^{k_{1}}[(l \\
& \left.+1)^{-a}, \Gamma a\right]\left.\right|_{a=1} ^{\infty} \\
& +2 \alpha \lambda \mu^{n} \sum_{k_{2}=0} \sum_{l=0}^{\infty} \frac{(-1)^{l+k_{2}} \Gamma(2 \alpha) \Gamma(n+1)}{k_{2} ! l ! \Gamma(2 \alpha-l) \Gamma\left(n-k_{2}+1\right)}\left(\frac{\sigma}{\mu}\right)^{k_{2}}\left(\frac{\partial}{\partial a}\right)^{k_{2}}[(l \\
& \left.+1)^{-a} \Gamma a\right]\left.\right|_{a=1}
\end{aligned}
$$

for $n=0,1,2,3, \ldots$

Putting $n=1$ and $n=2$ in the equation (19), the expression for $1^{\text {st }}$ moment (mean) and $2^{\text {nd }}$ moment are obtained as follows

$$
\begin{aligned}
& E(X)=\Gamma(\alpha+1) \sum_{l=0}^{\infty} \frac{(-1)^{l}(1-\lambda)\{\mu+\sigma C+\sigma \ln (l+1)\}}{(l+1) ! \Gamma(\alpha-l)} \\
&+\Gamma(2 \alpha+1) \sum_{l=0}^{\infty} \frac{(-1)^{l} \lambda\{\mu+\sigma C+\sigma \ln (l+1)\}}{(l+1) ! \Gamma(2 \alpha-l)}
\end{aligned}
$$

and

$E\left(X^{2}\right)$

$=\Gamma(\alpha$

$+1) \sum_{l=0}^{\infty} \frac{(-1)^{l}(1-\lambda)\left\{6 \mu^{2}+12 \mu \sigma C+12 \sigma(\mu+\sigma C) \ln (l+1)+6 \sigma^{2} C^{2}+\pi^{2} \sigma^{2}+6 \sigma^{2}\left((\ln (l+1))^{2}-\ln (l+1)\right)\right\}}{\{6(l+1) ! \Gamma(\alpha-l)\}}$

$+\Gamma(2 \alpha$

$+1) \sum_{l=0}^{\infty} \frac{(-1)^{l} \lambda\left\{6 \mu^{2}+12 \mu \sigma C+12 \sigma(\mu+\sigma C) \ln (l+1)+6 \sigma^{2} C^{2}+\pi^{2} \sigma^{2}+6 \sigma^{2}\left(\left(\ln (l+1)^{2}-\ln (l+1)\right)\right\}\right.}{\{6(l+1) ! \Gamma(2 \alpha-1)\}}$

Where $C$ is Euler's constant. In particular $\Gamma^{/}(1)=-C, \Gamma^{/}(2)=1-C, \Gamma^{/ /}(1)=C^{2}+$ $\frac{\pi^{2}}{6}$ and $C=\lim _{s \rightarrow \infty}\left(\sum_{m=1}^{s} \frac{1}{m}-\ln s\right)=0.577215$

And $V(X)=E\left(X^{2}\right)-\{E(X)\}^{2}$

\section{Moment Generating Function of TEGD:}

If $\mathrm{X}$ has a TEGD then the moment generating function of $\mathrm{X}$, say $M_{x}(t)$ is, obtained as

$$
\begin{aligned}
M_{X}(t)=\alpha e^{t \mu} & (1-\lambda) \sum_{l=0}^{\infty} \frac{(-1)^{l} \Gamma \alpha \Gamma(1-t \sigma)}{l ! \Gamma(\alpha-l)}(1+l)^{t \sigma-1} \\
& +2 \lambda \alpha e^{t \mu} \sum_{l=0}^{\infty} \frac{(-1)^{l} \Gamma(2 \alpha) \Gamma(1-t \sigma)}{l ! \Gamma(2 \alpha-l)}(1+l)^{t \sigma-1}
\end{aligned}
$$




\section{Random Number Generation and Parameter Estimation of TEGD:}

Using the method of inversion we have generated random numbers for the TEGD as

$$
1-\left\{1-\exp \left(-\exp \left(-\frac{x-\mu}{\sigma}\right)\right)\right\}^{\alpha}\left[1-\lambda+\lambda\left\{1-\exp \left(-\exp \left(-\frac{x-\mu}{\sigma}\right)\right)\right\}^{\alpha}\right]=u
$$

Where $u \sim U(0,1)$. This yields

$$
x=\mu-\sigma \ln \left[-\ln \left[1-\left\{1-\left\{\frac{(1+\lambda)-\sqrt{(1+\lambda)^{2}-4 \lambda u}}{2 \lambda}\right\}\right\}^{\frac{1}{\alpha}}\right]\right]
$$

Random number are generated by using (21), where the parameters $\mu, \boldsymbol{\sigma}, \alpha$ and $\lambda$ are known. The maximum likelihood estimates (MLE) of the parameters that are inherent within the TEGD function is given by the following:

$$
\begin{aligned}
L=\left(\frac{\alpha}{\sigma}\right)^{n} \prod_{i=1}^{n} & {[1} \\
& \left.-\exp \left\{-\exp \left(-\frac{x_{i}-\mu}{\sigma}\right)\right\}\right]^{\alpha-1} \exp \left\{-\exp \left(-\frac{x_{i}-\mu}{\sigma}\right)\right\} \exp \left(-\frac{x_{i}-\mu}{\sigma}\right)[1 \\
& \left.-\lambda+2 \lambda\left[1-\exp \left\{-\exp \left(-\frac{x_{i}-\mu}{\sigma}\right)\right\}\right]^{\alpha}\right] \\
\log L=n \log \alpha & -n \log \sigma \\
+ & (\alpha-1) \sum_{i=1}^{n} \log \left[1-\exp \left\{-\exp \left(-\frac{x_{i}-\mu}{\sigma}\right)\right\}\right] \\
& -\sum_{i=1}^{n} \exp \left(-\frac{x_{i}-\mu}{\sigma}\right)-\sum_{i=1}^{n}\left(\frac{x_{i}-\mu}{\sigma}\right) \\
& +\sum_{i=1}^{n} \log \left[1-\lambda+2 \lambda\left\{1-\exp \left\{-\exp \left(-\frac{x_{i}-\mu}{\sigma}\right)\right\}\right\}^{\alpha}\right]
\end{aligned}
$$

The first derivative of (23) w.r.t the parameters $\mu, \alpha, \sigma$ and $\lambda$ are expressed in (24), (25), (26) \& (27) respectively as

$$
\begin{aligned}
& \frac{\partial \log L}{\partial \mu} \\
& =\frac{n}{\sigma}+\frac{\alpha-1}{\sigma} \sum_{i=1}^{n} \frac{\exp \left(-\frac{x_{i}-\mu}{\sigma}\right) \exp \left\{-\exp \left(-\frac{x_{i}-\mu}{\sigma}\right)\right\}}{1-\exp \left\{-\exp \left(-\frac{x_{i}-\mu}{\sigma}\right)\right\}}-\frac{1}{\sigma} \sum_{i=1}^{n} \exp \left(-\frac{x_{i}-\mu}{\sigma}\right) \\
& +\frac{2 \lambda \alpha}{\sigma} \sum_{i=1}^{n} \frac{\exp \left(-\frac{x_{i}-\mu}{\sigma}\right) \exp \left\{-\exp \left(-\frac{x_{i}-\mu}{\sigma}\right)\right\}\left[1-\exp \left\{-\exp \left(-\frac{x_{i}-\mu}{\sigma}\right)\right\}\right]^{\alpha-1}}{1-\lambda+2 \lambda\left[1-\exp \left\{-\exp \left(-\frac{x_{i}-\mu}{\sigma}\right)\right\}\right]^{\alpha}}
\end{aligned}
$$




$$
\begin{aligned}
& \frac{\partial \log L}{\partial \sigma} \\
& =-\frac{n}{\sigma}+\frac{\alpha-1}{\sigma^{2}} \sum_{i=1}^{n} \frac{\left(x_{i}-\mu\right) \exp \left(-\frac{x_{i}-\mu}{\sigma}\right) \exp \left\{-\exp \left(-\frac{x_{i}-\mu}{\sigma}\right)\right\}}{1-\exp \left\{-\exp \left(-\frac{x_{i}-\mu}{\sigma}\right)\right\}} \\
& +\sum_{i=1}^{n}\left(\frac{x_{i}-\mu}{\sigma^{2}}\right)\left\{1-\exp \left(-\frac{x_{i}-\mu}{\sigma}\right)\right\} \\
& +\frac{2 \alpha \lambda}{\sigma^{2}} \sum_{i=1}^{n} \frac{\left(x_{i}-\mu\right) \exp \left(-\frac{x_{i}-\mu}{\sigma}\right) \exp \left\{-\exp \left(-\frac{x_{i}-\mu}{\sigma}\right)\right\}\left[1-\exp \left\{-\exp \left(-\frac{x_{i}-\mu}{\sigma}\right)\right\}\right]^{\alpha-1}}{1-\lambda+2 \lambda\left[1-\exp \left\{-\exp \left(-\frac{x_{i}-\mu}{\sigma}\right)\right\}\right]^{\alpha}} \\
& \frac{\partial \log L}{\partial \alpha} \\
& =\frac{n}{\alpha}+\sum_{i=1}^{n} \log \left[1-\exp \left\{-\exp \left(-\frac{x_{i}-\mu}{\sigma}\right)\right\}\right] \\
& +2 \alpha \lambda \sum_{i=1}^{n} \frac{\log \left[1-\exp \left\{-\exp \left(-\frac{x_{i}-\mu}{\sigma}\right)\right\}\right]}{1-\lambda+2 \lambda\left[1-\exp \left\{-\exp \left(-\frac{x_{i}-\mu}{\sigma}\right)\right\}\right]^{\alpha}}
\end{aligned}
$$

and

$$
\frac{\partial \log L}{\partial \lambda}=\sum_{i=1}^{n} \frac{2\left[1-\exp \left\{-\exp \left(-\frac{x_{i}-\mu}{\sigma}\right)\right\}\right]^{\alpha}-1}{1-\lambda+2 \lambda\left[1-\exp \left\{-\exp \left(-\frac{x_{i}-\mu}{\sigma}\right)\right\}\right]^{\alpha}}
$$

Setting (24), (25), (26) \& (27) to zero and solving them simultaneously yields the maximum likelihood estimates of four parameters. A small Monte Carlo simulation experiment based on 1000 replications was conducted to evaluate the MLE's of the parameters of TEGD. We set the sample size at $n=1000$, the parameter $\alpha$ at $\alpha=2$ and $\lambda$ at $\lambda=0.6$. The location and scale parameter were fixed at $\mu=0$ and $\sigma=1$ respectively. The Monte Carlo simulation experiments are performed using the RProgramming language. We get the estimated value of the parameter and standard error as follows:

Table 1: Estimated values of $\mu, \sigma, \lambda \& \alpha$ and standard error of parameters

\begin{tabular}{|c|c|c|}
\hline Parameters & Estimated Values & Std. Error \\
\hline$\hat{\mu}$ & 0.09226249 & 0.16522530 \\
$\hat{\sigma}$ & 1.55016699 & 0.08998573 \\
$\hat{\lambda}$ & 0.58537495 & 0.19632186 \\
$\hat{\alpha}$ & 2.19048044 & 0.47983809 \\
\hline
\end{tabular}

Loglikelihood: $-1072.76 \quad$ AIC: $2153.521 \quad$ BIC: 2173.152 


\section{Reliability Analysis of TEGD:}

The Reliability Function $R(t)$, is defined by $R(t)=1-F(t)$ and for TEGD it is given as

$R(t)=\left[1-\exp \left\{-\exp \left(-\frac{x-\mu}{\sigma}\right)\right\}\right]^{\alpha}\left[1-\lambda+\lambda\left\{1-\exp \left\{-\exp \left(-\frac{x-\mu}{\sigma}\right)\right\}\right\}^{\alpha}\right]$

Using (*) we can expressed Reliability Function of TEGD can be written in mixture form as

$R(t)=(1-\lambda) \sum_{j=0}^{\infty} \frac{(-1)^{j} \Gamma(\alpha+1)}{\Gamma(\alpha-j+1)} \frac{v^{j}}{j !}+\lambda \sum_{j=0}^{\infty} \frac{(-1)^{j} \Gamma(2 \alpha+1)}{\Gamma(2 \alpha-j+1)} \frac{v^{j}}{j !}$

Where $v=\exp \left\{-\exp \left(-\frac{x-\mu}{\sigma}\right)\right\}$

\section{Hazard Rate Function:}

The hazard rate function or instantaneous failure rate, which is an important quality characterizing life phenomenon defined by $h(t)=\frac{f(t)}{1-F(t)}$

The hazard rate function for TEGD is given by

$=\frac{\alpha}{\sigma} \frac{\exp \left(-\frac{x-\mu}{\sigma}\right) \exp \left\{-\exp \left(-\frac{x-\mu}{\sigma}\right)\right\}\left[1-\lambda+2 \lambda\left\{1-\exp \left\{-\exp \left(-\frac{x-\mu}{\sigma}\right)\right\}\right\}^{\alpha}\right]}{\left[1-\exp \left\{-\exp \left(-\frac{x-\mu}{\sigma}\right)\right\}\right]\left[1-\lambda+\lambda\left\{1-\exp \left\{-\exp \left(-\frac{x-\mu}{\sigma}\right)\right\}\right\}^{\alpha}\right]}$

Using (*) we can expressed Hazard Rate Function of TEGD can be written in mixture form as

$h(t)=\frac{\alpha}{\sigma}\left(\frac{v}{1-v}\right) \log \left(\frac{1}{v}\right) \frac{\left[1-\lambda+2 \lambda \sum_{j=0}^{\infty} \frac{(-1)^{j} \Gamma(\alpha+1)}{\Gamma(\alpha-j+1)} \frac{v^{j}}{j !}\right]}{\left[1-\lambda+\lambda \sum_{j=0}^{\infty} \frac{(-1)^{j} \Gamma(\alpha+1)}{\Gamma(\alpha-j+1)} \frac{v^{j}}{j !}\right]}$

where $v=\exp \left\{-\exp \left(-\frac{x-\mu}{\sigma}\right)\right\}$

\section{Application of TEGD to Experimental Data}

Survivability of a theoretical statistical model depends on its practical application. That is why, we provide an application to real data set to illustrate the importance of TEGD. Here we work with water quality data using some water quality parameters. Data for water quality parameters have been taken from the Department of Chemistry, Gauhati University. Various water quality parameters were estimated for the project entitled "Assessment of Toxic Element in Water of Semi-Under Area of Assam and Investigation of the Disease Related Contaminants" during the $1^{\text {st }}$ March to $31^{\text {st }}$ October, 2009 for three administration sub-divisions of Nogaon district of Assam, India. Since the probability distribution of estimated data of iron (Fe) follows the theoretical TEG distribution. Therefore, to test the goodness of fit of this distribution, estimated 
values of iron (Fe) have been applied in TEG distribution. Here we compute the MLE's of the parameters and the goodness-of-fit statistics for this distribution are compared with EGD and GD, to show the importance of new model. All computations have been carried out using R-Programming language.

The graphical representations of Reliability Function \& Hazard Rate Function of TEGD for known values of different parameters are presented in Fig. 3 \& Fig. 4 respectively.

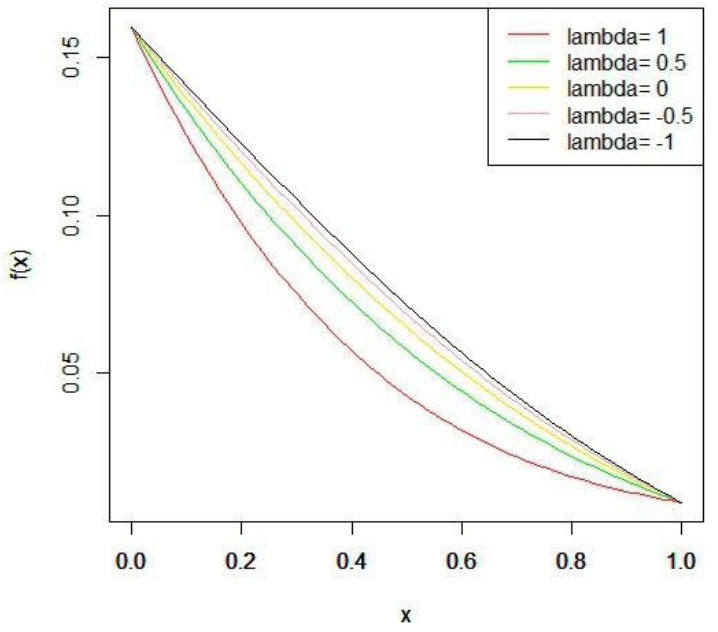

Fig. 3: Reliability Function of TEGD for assumed values of $\alpha=2, \mu=0, \sigma=$ $1 \& \lambda=1,0.5,0,-0.5, \&-1$

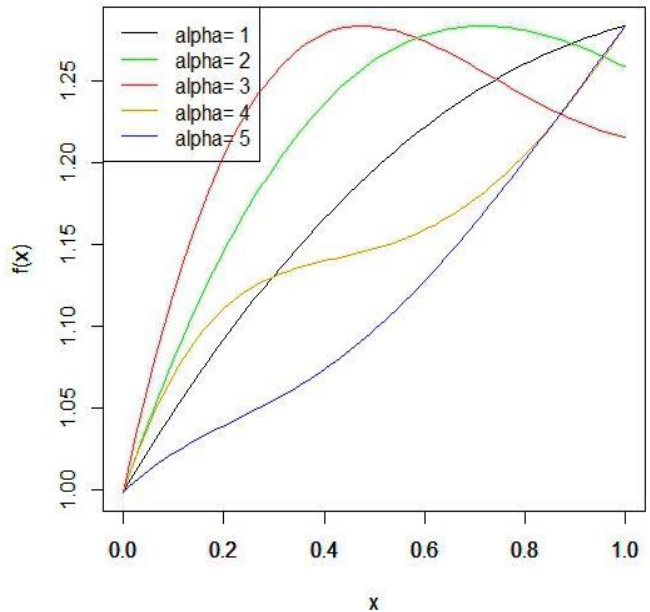

Fig. 4: Hazard Rate Function of TEGD for assumed values of $=0, \sigma=1$, $\lambda=0.8$ and $\alpha=1,2,3,4$ and 5

To compare TEGD with EGD \& GD, we consider some criteria like $-2 l($.$) : where l$ is the maximum value of log-likelihood function, AIC (Akaike Information Criterion), CAIC (Corrected Akaike Information Criterion) and BIC (Bayesian Information Criterion) for the data set. In general the better fit of the distribution corresponds to the smaller value of the statistics $-2 l($.$) , AIC, CAIC and BIC.$

Table 2: Estimated parameters of the TEGD, EGD \& GD for the water quality data set

\begin{tabular}{|l|c|c|c|}
\hline \multicolumn{1}{|c|}{ Distribution } & Estimated Parameter & Standard Error & $-l(. ; x)$ \\
\hline & $\hat{\mu}=0.2599105$ & 0.1807813 & \\
Transmuted Exponentiated & $\hat{\sigma}=0.1856675$ & 0.2130491 & \multirow{2}{*}{39.7011} \\
Gumbel Distribution (TEGD) & $\hat{\alpha}=0.1813843$ & 0.2449015 & \\
& $\hat{\lambda}=-0.5304026$ & 1.1518608 & \\
\hline \multirow{2}{*}{ Exponentiated Gumbel } & $\hat{\mu}=0.4920412$ & 0.2745998 & \multirow{2}{*}{39.7599} \\
Distribution (EGD) & $\hat{\sigma}=0.3220115$ & 0.6995206 & \\
\hline \multirow{2}{*}{ Gumbel Distribution (GD) } & $\hat{\alpha}=0.2909981$ & 0.6550300 & \\
& $\hat{\mu}=1.0639522$ & 0.3071901 & \multirow{2}{*}{40.8023} \\
\hline
\end{tabular}


Table 3: Comparison of TEGD, EGD \& GD

\begin{tabular}{|l|c|c|c|c|}
\hline \multicolumn{1}{|c|}{ Distribution } & $-2 l()$. & AIC & CAIC & BIC \\
\hline $\begin{array}{l}\text { Transmuted Exponentiated } \\
\text { Gumbel Distribution (TEGD) }\end{array}$ & 79.4022 & 85.5198 & 87.1198 & 88.4071 \\
\hline $\begin{array}{l}\text { Exponentiated Gumbel } \\
\text { Distribution (EGD) }\end{array}$ & 79.5198 & 86.6045 & 87.5278 & 89.7233 \\
\hline Gumbel Distribution (GD) & 81.6047 & 87.4023 & 87.8468 & 93.0071 \\
\hline
\end{tabular}

The observed values of-2l(.), AIC, CAIC and BIC for TEGD, EGD \& GD from Table 3, it is clear that Transmuted Exponentiated Gumbel Distribution (TEGD) gives lower values than that of Exponentiated Gumbel Distribution (EGD) and Gumbel Distribution (GD). Hence TEGD leads to a better fit than the other two distributions.

\section{Conclusion}

The TEGD has been generated and their parameters are estimated. The TEGD is applied to water quality data and it is compared with EGD \& GD and leads to better fit than the other two distributions. Hence the new TEGD can be applied to environmental science for better modeling.

\section{References}

1. Afify, A.Z., Nofal, Z.M. and Yousof, H.M. (2015).The Transmuted Weibull Lomax Distribution: Properties and Application. Pak.J.Stat.Oper.res., 11, 135152.

2. Ahmad, A., Ahmad, S.P. and Ahmad, A. (2014). Transmuted Inverse Rayleigh Distribution: A Generalization of the Inverse Rayleigh Distribution. Mathematical Theory and Modeling, 4, 90-98.

3. Aryal, G.R. and Tsokos, C.P. (2009). On the transmuted extreme value distribution with application. Nonlinear Analysis: Theory, Methods and Application, 71, 1401-1407.

4. Aryal, G.R. (2013). Transmuted Log-Logistic Distribution. Journal of Statistics Application and Probability, 2, 11-20.

5. Ashour, S.K. and Eltehiwy, M.K. (2013). Transmuted Lomax Distribution. American Journal of Applied Mathematics and Statistics, 1, 121-127.

6. Ashour, S.K. and Eltehiwy, M.K. (2013). Transmuted Exponentiated Modified Weibull Distribution. International Journal of Basic and Applied Sciences, 2, 258269.

7. Cardeiro, G.M., Nadarajah, S. and Ortega, E.M.M. (2012). The Kumaraswamy Gumbel distribution. Stat Methods Appl, 21, 139-168.

8. Elbatal, I. (2013). Transmuted Modified Inverse Weibull Distribution: A Generalization Of The Modified Inverse Weibull Probability Distribution. International Journal of Mathematical Archeive, 4,117-129. 
9. Khan, M.S. and King, R. (2013). Transmuted Generalized Inverse Weibull Distribution. Journal of Applied Statistical Science, 20, 15-31.

10. Kotz, S and Nadarajah, S. (2000). "Extreme value Distributions: Theory and Applications". London: Imperical College Press.

11. Lucena, S.E.F., Silva, A.H.A. and Cordeiro, G.M. (2015). The Transmuted Generalized Gamma Distribution: Properties and Application. Journal of Data Science, 13, 187-206.

12. Mahmoud, M.R. and Mandouh, R.M. (2013). Transmuted Frechet Distribution. Journal of Applied Science and Research, 9, 5553-5561.

13. Merovci, F. (2013). Transmuted Rayleigh Distribution. Austrian Journal of Statistics, 42, 21-32.

14. Nadarajah, S. (2006). The exponentiated Gumbel distribution with climate application. Environmetrics, 17, 13-23.

15. Prudnikov A.P., Brychkov Yu. A. and Marichev O.I. (1986). Integrals and Series, Vol.1, Gordon and Breach Science Publishers: Amsterdam.

16. Shaw, W.T and Buckley, I.R.C. (2007). The Alchemy of Probability Distributions: Beyond Gram-Charlier \& Cornish-Fisher Expansions, and SkewNormal or Kurtotic-Normal Distributions. Research report. 